\title{
Leigh syndrome caused by mutations in the flavoprotein (Fp) subunit of succinate dehydrogenase (SDHA)
}

\author{
R Horváth, A Abicht, E Holinski-Feder, A Laner, K Gempel, H Prokisch, H Lochmüller, T Klopstock, \\ $M$ Jaksch
}

See end of article for authors' affiliations

Correspondence to:

Dr Rita Horváth, Metabolic

Disease Centre Munich-

Schwabing, Institutes of

Clinical Chemistry,

Molecular Diagnostics and

Mitochondrial Genetics;

Academic Hospital

Schwabing, Kölner Platz 1,

80804 Munich, Germany;

Rita.Horvath@

Irz.uni-muenchen.de

Received 8 March 2005

In revised form

27 April 2005

Accepted 19 May 2005
J Neurol Neurosurg Psychiatry 2006;77:74-76. doi: 10.1136/jnnp.2005.067041

Detailed clinical, neuroradiological, histological, biochemical, and genetic investigations were undertaken in a child suffering from Leigh syndrome. The clinical symptoms started at age five months and led to a severe progressive neurodegenerative disorder causing epilepsy, psychomotor retardation, and tetraspasticity. Biochemical measurement of skeletal muscle showed a severe decrease in mitochondrial complex II. Sequencing of SDHA revealed compound heterozygosity for a nonsense mutation in exon 4 (W119X) and a missense mutation in exon 3 (A83V), both absent in normal controls. In six additional patients - five with Leigh or Leigh-like syndrome and one with neuropathy and ataxia associated with isolated deficiency of complex II-mutations in SDHA were not detected, indicating genetic heterogeneity.
D efects of the human succinate dehydrogenase (SDH) are associated with a highly variable clinical presentation, ranging from early onset encephalomyopathies to tumour susceptibility in adults. ${ }^{2} \mathrm{SDH}$ is made up of four subunits-two soluble proteins, the flavoprotein (Fp, SdhA) and the Fe-S protein ( $\mathrm{SdhB}$ ) which are anchored to the inner membrane by the subunits SdhC and SdhD. ${ }^{12}$ Pathogenic mutations in the SDHA gene have so far rarely been described..$^{3-7}$

\section{CASE REPORT}

The nine year old girl was first child of non-consanguineous German parents. Family history was negative. The patient was born healthy at 38 weeks after normal pregnancy (weight $2700 \mathrm{~g}$, length $49 \mathrm{~cm}$, head circumference $34 \mathrm{~cm}$ ). At five months of age developmental delay with axial hypotonia and gaze fixation difficulties were noted. At eight months of age (weight $7780 \mathrm{~g}$ (50th centile), length $65 \mathrm{~cm}$ (3rd centile), head circumference: $41 \mathrm{~cm}$ (3rd centile)), neurological examination showed generalised muscular hypotonia with axial predominance. She could not fix her gaze and bilateral horizontal nystagmus was noted. Deep tendon reflexes were bilaterally increased, with Achilles clonus and Babinski sign. Neither grasping nor eye contact was possible. Focal and generalised seizures were noted clinically. Heart and abdominal organs were normal. Electroencephalography (EEG) showed slow, irregular basic activity and focal epileptic signs with secondary generalisation and hypsarrythmia. Routine laboratory indices were normal except for mildly increased lactate (in serum $2.9 \mathrm{mmol} / \mathrm{l}$ (normal 0.5-2.2); in CSF $2.7 \mathrm{mmol} / \mathrm{l}($ normal <2)) and creatine kinase $(170 \mathrm{U} / \mathrm{l}($ normal <100)). EEG, electronystagmography, and visual evoked potentials were normal.

At the age of three years (length $83 \mathrm{~cm}$ (<3rd centile), weight $9550 \mathrm{~g}$ ( $<3 \mathrm{rd}$ centile), head circumference $45 \mathrm{~cm}$ $(<3$ rd centile)) the child was alert, but not communicating.
Horizontal nystagmus, tetraspasticity with brisk deep tendon reflexes, Achilles clonus, and Babinski sign were encountered. EEG showed epileptic activity provoked by photostimulation and myoclonic jerks. BNS (Blitz-Nick-Salaam) cramps were noted.

Over recent years the child has shown an arrest of psychomotor functions with recurrent seizures and further development of severe contractures. At the current age of nine years she is tetraparetic, unable to speak, has complete visual loss, and has seizures without involvement of other organs. A muscle biopsy was done at age of nine years because of possible prenatal consequences.

\section{METHODS}

Morphology and biochemistry of skeletal muscle

Serial cross sections $(8 \mu \mathrm{m})$ were obtained for histochemical stains according to standard procedures. Activities of the respiratory chain (RC) complexes I-IV were determined in skeletal muscle of the patient as described. ${ }^{8}$

\section{DNA analysis}

DNA and RNA extraction was done according to standard purification protocols (Qiagen, Hildesheim, Germany). Direct sequencing of fibroblast cDNA as well as the promoter region was carried out according to Parfait et al. ${ }^{4}$ We constructed intronic primers for amplification of the nuclear gene (online only material). Polymerase chain reaction (PCR) for sequencing the nuclear gene was done by using the HotStar Taq kit (Qiagen, Hildesheim, Germany) with $50^{\circ} \mathrm{C}$ annealing temperature.

Denaturing high performance liquid chromatography Denaturing high performance liquid chromatography (DHPLC) analysis was carried out with the PCR product of

Abbreviations: DHPLC, denaturing high performance liquid chromatography; RC, respiratory chain; SDH, succinate dehydrogenase 
exon 3 of SDHA as described ${ }^{9}$ on an automated HPLC device equipped with a DNA separation column (WAVE $^{\mathrm{TM}}$ DNA Fragment Analysis System: Transgenomic, San Jose, California, USA). All fragments were separated by an universal gradient, starting at $40 \%$ and ending at $70 \%$ of buffer B (Transgenomic), over a period of seven minutes at temperatures of $56^{\circ}, 58^{\circ}$, and $59^{\circ} \mathrm{C}$.

\section{Immunoblotting}

Twenty five micrograms of protein from skeletal muscle homogenates were loaded on $12 \%$ polyacrylamide gels. The following antibodies were used according to the recommendations of the supplier: mouse monoclonal anti-SDHA antibody (anti-complex II Fp $70 \mathrm{kDa}$, Molecular Probes, 1:2000), anti-chicken actin (Developmental Studies Hybridoma Bank, University of Iowa, 1:2000) and secondary anti-mouse IgG coupled to HRP (Dako) 1:2000. Signals were visualised with enhanced chemiluminescence (ECL plus; Amersham Pharmacia Biotech) according to the manufacturer's recommendations.

\section{RESULTS}

\section{Magnetic resonance imaging}

Brain magnetic resonance imaging (MRI) at age eight months showed bilateral cortical atrophy with massive T2 weighted signal intensities in the basal ganglia involving the thalamus, pallidum, substantia nigra, nucleus ruber, and nucleus caudatus; however, the brain stem and the cerebellum were normal.

At age three years, cortical atrophy and ventricular enlargement had markedly progressed. The T2 weighted signal hyperintensities in the putamen and nucleus caudatus were still present; however, the bilateral thalamus lesions had disappeared. Defect myelination was described predominantly in the periventricular white matter. Cerebellum and pons were normal.

\section{Morphology and biochemistry of skeletal muscle} Muscle biopsy of the left tibialis anterior muscle showed normal results on light and electron microscopy. Histochemistry for SDH showed equal staining of all fibres. Biochemical measurements of the respiratory chain enzymes revealed a significantly decreased activity of complex II (3.7 U/gNCP; normal range 16 to $42 \mathrm{U} / \mathrm{gNCP}, 23 \%$ residual activity) but no abnormalities in the activities of other RC enzymes (pyruvate dehydrogenase and citrate synthase).

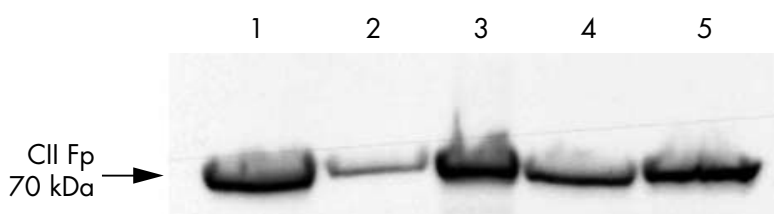

Figure 1 Upper panel: Immunoblotting with anti-SDHA antibody (CII $\mathrm{Fp}$ ) showed a significantly diminished signal in the muscle homogenate from the patient (lane 2) compared with normal controls (lanes 1, 3, 4, and 5), thus indicating a reduced steady state level of SDHA. Lower panel: Incubation of the same blot with anti-actin antibody revealed equal loading.

\section{DNA analysis}

Direct sequencing of SDHA revealed two compound heterozygous mutations in the patient: one nonsense mutation in exon 4 (W119X) inherited from the father, and a missense mutation in exon 3 (A83V) inherited from the mother. Both mutations were also present in the patient's cDNA; however, the W119X allele was difficult to detect, possibly because of nonsense mediated decay. A83V was not present in 100 normal control DNA samples screened by WAVE. Sequencing of all exons, the exon-intron boundaries, and the SDHA promoter region revealed several polymorphisms, present in the patient as well as in control DNA samples (online-only material).

By sequencing the cDNA of SDHA, we detected an additional cDNA species expressed by the previously described SDHA pseudogene on chromosome 3 that showed a few differences compared with the "true" SDHA gene on chromosome 5, including a frameshift mutation leading to a stop codon in exon 2.

\section{Immunoblotting}

Immunoblotting with anti-SDHA antibody (CII Fp) showed a significantly diminished signal in the patient compared with controls, thus indicating a reduced steady state level of this protein. As a control for the equal loading, an immunodetection of the same blot was performed using actin antibody (fig l).

\section{DISCUSSION}

Isolated complex II deficiency is a very rare condition, occurring in approximately $2-4 \%$ of all respiratory chain enzyme deficiencies. ${ }^{4}$ Mutations of SDHA have been reported in only five families so far. Three of these presented with autosomal recessive Leigh syndrome, ${ }^{3-5}$ and one child died suddenly at the age of five months from a severe deterioration of neuromuscular, cardiac, and hepatic symptoms after an intermittent infection. ${ }^{6}$ All of these children were carrying missense mutations in the C-terminal domain. In the fifth family, two siblings suffered from late onset optic atrophy, ataxia, and myopathy, and a heterozygous mutation was identified, suggesting a possible role of SDHA mutations in autosomal dominant neurodegenerative disorders. ${ }^{7}$ Further studies showed that several patients with complex II deficiency do not have mutations of SDHA. This suggested a role of additional nuclear genes involved in synthesis, assembly, or maintenance of $\mathrm{SDH}^{4}$ Interestingly, dominant mutations of the three other SDH subunit genes ( $S D H B$, $S D H C$, and $S D H D$ ) were only described in association with tumours, such as hereditary paraganglioma and abdominal phaeochromocytoma. ${ }^{10}$

The biochemical complex II defect in our patient was confirmed by a significantly reduced level of SDHA protein in muscle and compound heterozygote mutations in the SDHA gene. Although the activity of complex II was markedly reduced (23\% rest activity), SDH was still detectable by histochemistry. We hypothesise that partial SDH decrease may be overlooked if histochemistry only is performed. The heterozygote missense mutation $(\mathrm{A} 83 \mathrm{~V})$ inherited from the mother occurred in a region of the protein which is conserved over a wide range of species (online-only material). It was not found in more than 200 control alleles. W119X is the first stop mutation described in SDHA; all other previously described mutations were missense mutations. Both parents are healthy and carry one of the mutations heterozygously, supporting a recessive trait in this family.

Parfait et al ${ }^{4}$ reported two almost identical SDHA gene copies encoding SDHA (Chr 5pl5 and 3q29) with preserved genomic organisation ( 15 exons for both copies). The SDHA copy on chromosome $3 q 29$ contains a l base pair (bp) 
Table 1 Clinical summary of six patients with isolated complex II deficiency not showing SDHA mutations

\begin{tabular}{|c|c|c|c|c|}
\hline Patient & Age of onset & Family history & Clinical symptoms & $\begin{array}{l}\text { Complex II activity } \\
\text { (\% lower normal range) }\end{array}$ \\
\hline AJ (female) & Early infancy & Sporadic & Severe muscular hypotonia & $50 \%$ \\
\hline LS (female) & $5 \mathrm{~m}$ & Sporadic & $\begin{array}{l}\text { Muscular hypotonia, } \\
\text { psychomotor retardation }\end{array}$ & $60 \%$ \\
\hline MC (female) & $1 y$ & Sporadic & $\begin{array}{l}\text { Typical Leigh syndrome, } \\
\text { hypertrophic cardiomyopathy }\end{array}$ & $25 \%$ \\
\hline MR (male) & Early infancy & Sporadic & $\begin{array}{l}\text { Severe muscular hypotonia, } \\
\text { psychomotor retardation }\end{array}$ & $30 \%$ \\
\hline TS (male) & $3 \mathrm{~m}$ & $\begin{array}{l}\text { Affected sibling, } \\
\text { consanguineous } \\
\text { parents }\end{array}$ & $\begin{array}{l}\text { Muscular hypotonia, } \\
\text { respiratory insufficiency, } \\
\text { psychomotor retardation, } \\
\text { epilepsy }\end{array}$ & $40 \%$ \\
\hline BR (male) & $3 y$ & Sporadic & Ataxia, neuropathy & $60 \%$ \\
\hline
\end{tabular}

deletion in exon 2 which is predicted to cause a frameshift and premature termination of the amino acid chain. Thus the SDHA copy on Chr 3q29 probably represents a pseudogene. However, by analysing cDNA as described ${ }^{4}$ we found that both SDHA copies are expressed, which is in contrast to a previous report. ${ }^{3}$ Thus by screening cDNA only, heterozygous mutations might easily be missed as they represent $25 \%$ (one in four alleles) or fewer. This may cast doubt on an SDHA mutation detected by cDNA sequencing which was thought to have a "dominant" effect. ${ }^{71}$ We therefore designed intronic primers to specifically amplify and sequence the SDHA gene on chromosome 5.

Six additional patients of our cohort similarly showed a severe isolated complex II deficiency-five presented with Leigh or Leigh-like syndrome and one with neuropathy and ataxia (table 1).

No pathogenic mutations in SDHA were found, suggesting genetic heterogeneity that confirms previous studies. ${ }^{4}$ Further investigation using cells or tissues of these patients are warranted in order to identify additional genes and thus far unknown mechanisms involved in the maintenance and assembly of mitochondrial complex II.

\section{ACKNOWLEDGEMENTS}

This work is supported by grants from the Deutsche Forschungsgemeinschaft Ja802/2-1 (MJ), the Ernst and Berta Grimmke Stiftung (MJ, RH), and the German National Genome Network (German Ministry for Education and Research OIGRD4ll; $\mathrm{HP}$ ).

\section{Authors' affiliations}

R Horváth, K Gempel, M Jaksch, Metabolic Disease Centre MunichSchwabing, Institutes of Clinical Chemistry, Molecular Diagnostics and Mitochondrial Genetics, Academic Hospital Schwabing, Munich, Germany
A Abicht, $\mathbf{H}$ Lochmüller, Friedrich-Baur-Institute and Department of Neurology, Ludwig-Maximilians University, Munich

T Klopstock, Department of Neurology, Ludwig-Maximilians University E Holinski-Feder, A Laner, Medical Genetic Centre, Munich H Prokisch, GSF National Research Centre, Institute of Human Genetics, Munich

Competing interests: none declared

\section{REFERENCES}

1 Rustin P, Munnich A, Rotig A. Succinate dehydrogenase and human diseases: new insights into a well-known enzyme. Eur J Hum Genet 2002; 10:289-91.

2 Rustin P, Rotig A. Inborn errors of complex II - unusual human mitochondrial diseases. Biochim Biophys Acta 2002;1553:117-22.

3 Bourgeron T, Rustin P, Chretien D, et al. Mutation of a nuclear succinate dehydrogenase gene results in mitochondrial respiratory chain deficiency. Nat Genet 1995;11:144-9.

4 Parfait B, Chretien D, Rotig A, et al. Compound heterozygous mutations in the flavoprotein gene of the respiratory chain complex II in a patient with Leigh syndrome. Hum Genet 2000; 106:236-43.

5 Goto Y, Murayama K, Hirawake H, et al. Molecular genetics of 5 patients with succinate dehydrogenase deficiency [abstract]. Am J Hum Genet 1998;(suppl 63):A362.

6 Van Coster R, Seneca S, Smet J, et al. Homozygous Gly555Glu mutation in the nuclear-encoded $70 \mathrm{kDa}$ flavoprotein gene causes instability of the respiratory chain complex II. Am J Med Genet 2003;120A:13-18.

7 Birch-Machin MA, Taylor RW, Cochran B, et al. Late-onset optic atrophy, ataxia, and myopathy associated with a mutation of a complex II gene. Ann Neurol 2000;48:330-5.

8 Fischer JC, Ruitenbeek W, Gabreels FJ, et al. A mitochondrial encephalomyopathy: the first case with an established defect at the level of coenzyme Q. Eur J Pediatr 1986;144:441-4.

9 Holinski-Feder E, Muller-Koch Y, Fried W, et al. DHPLC mutation analysis of the hereditary nonpolyposis colon cancer (HNPCC) genes hMLHI and hMSH2. J Biochem Biophys Methods 2001;47:21-32.

10 Astuti $D$, Morris M, Krona C, et al. Investigation of the role of SDHB inactivation in sporadic phaeochromocytoma and neuroblastoma. $\mathrm{Br} J$ Cancer 2004;91:1835-41.

11 Taylor RW, Birch-Machin MA, Schaefer J, et al. Deficiency of complex II of the mitochondrial respiratory chain in late-onset optic atrophy and ataxia. Ann Neurol 1996;39:224-32. 